\title{
Performance Comparison of Various Filters for Removing Poisson Noise, Exponential Noise, Multiplicative Noise and Erlang Noise
}

\author{
PrashantDwivedy*, Anjali Potnis**, Madhuram Mishra***, ShahbazSoofi**** \\ *(Department of Digital Communication Engineering, NITTTR, Bhopal-462002 \\ ** (Department of Digital Communication Engineering, NITTTR, Bhopal - 462002 \\ *** (Department of Digital Communication Engineering, NITTTR, Bhopal - 462002 \\ **** (Department of Digital Communication Engineering, NITTTR, Bhopal - 462002
}

\begin{abstract}
In Digital images there are many possibilities that images are prone to variety of noises. Therefore image denoising is to be an essential part for image reconstruction process. Generally noise in image gets introduced during acquisition, transmission, reception and storage \& retrieval processes. A detailed analysis of the various noises which corrupt an image is included in this paper. Removal of noises is done using various filters. To obtain significant results various filters have been proposed to remove these noises from images and finally which filter is most suitable to remove a particular noise is seen using various measurement parameters including Mean Square Error and Peak Signal to Noise Ratio.
\end{abstract}

Keywords: Poisson Noise, Multiplicative Noise, Erlang Noise, Exponential Noise, PSNR, MSE.

\section{INTRODUCTION}

When the digital images are processed using digital computers then removal or de-noising of noise is very essential [1]. In case of image noise is an unwanted signal which gives change in visibility of any image and occurs usually due to thermal or electrical signals such as from sensors or environmental conditions. The main problem at hand is removing the noise of an image while preserving its main features (edges, textures, colors, contrast, etc.) This has been regularly extensively investigated over the last two decades and numerous types of approaches have been developed. There are mainly two domain processes available for the restoring the image, first one is spatial domain and second one is frequency domain. In the spatial domain filtering action is done by operating on the pixel of the digital image directly for restoring the image. On the other hand filtering action is done by in frequency domain by mapping spatial domain into frequency domain of the image function by taking Fourier transform of the image function. After the filtering, in order to determine the restored image we have to re map the image into spatial domain by taking inverse Fourier transform. Noise may be generallyPoisson Noise, Multiplicative Noise,Erlang Noise and Exponential Noise. In this paper first image is taken and some noise is added to image to make it a noisy image and then noisy image is decomposed by filters. It becomes very important to de-noise the image before applying to different applications [2].The principle approach of image de-noising is filtering. Various filters are used to remove noise such as mid- point filters, median filters, mean filters etc. The image quality is measured by various performance parameters like the peak signal to noise ratio (PSNR) and mean square error (MSE) [3].

\section{NOISE MODELS}

The result of error in image acquisition process [4] that results in pixel values that do not reflect true intensities of the real picture is called noise. Using probability density functions we can define a set of noise models. The most occurring noises in digital images are Poisson noise, Exponential noise, Multiplicative noise, and Erlang noise or Gamma noise. Following, these noises are discussed at stretch.

\subsection{Poisson Noise}

Poisson noise or Shot noise is a type of electronic noise which can be modeled by a Poisson process. A Poisson model assumes that each pixel $x$ of an image $f(x)$ is drawn from a Poisson distribution of parameter $\lambda=\mathrm{f}(\mathrm{x})$ where $\mathrm{f}(0)$ is the clean intensity image to recover.

$$
P(f(x=k))=\left(\lambda^{k} e^{-\lambda}\right) / k !(1)
$$




\subsection{Exponential Noise}

This noise is modeled on the exponential probability distribution which is a special case of erlang with $\mathrm{b}=1$.

where $\mathrm{a}>0$.

$$
P(z)=\left\{\begin{array}{ll}
a e^{-z} \text { for } z & \geq 0 \\
0 & \text { for } z<0
\end{array}(2)\right.
$$

\subsection{Multiplicative Noise}

Multiplicative noise refers to an unwanted random signal that gets multiplied into some relevant signal during capture, transmission, or other processing [7]. An important example is the speckle noise. Generalized model of the multiplicative noise is described by $g(n, m)=f(n, m) * u(n, m)+k(n, m)(3)$

Where, $\mathrm{g}(\mathrm{n}, \mathrm{m})$ is the observed image, $\mathrm{u}(\mathrm{n}, \mathrm{m})$ is the multiplicative component and $\mathrm{k}(\mathrm{n}, \mathrm{m})$ is the additive component of the speckle noise [8]. Here $n$ and $m$ denote the axial and lateral indices of the image samples.

\subsection{Erlang Noise}

The PDF of Erlang noise [10] is given by

$$
P(z)= \begin{cases}\frac{a^{b} z^{b-1}}{(b-1) !} e^{-a z} & \text { for } z \geq 0 \\ 0 & \text { for } z<0\end{cases}
$$

\section{FILTERING TECHNIQUES}

Removal of noise from the degraded image is done by filtering. There are two types of filtering techniques [13]. First one being spatial filtering and second one is frequency filtering. Spatial filtering is the filtering operations that are performed directly on the pixels of image. In Spatial Domain the filtering operation [14] is done by convolving the image pixels with the pixels of the mask. A mask is a small sub image, often of size $3 \times 3$ pixels. The mask size is varied according to the requirement. These include the following classes of filters

- Mean filters

- Order statistics filters

- $\quad$ Adaptive filters

\subsection{Geometric Mean Filter}

The working of a geometric mean filter is same as the arithmetic mean filter; the only difference is that instead of taking the arithmetic mean the geometric mean is taken. The restored image is given by the expression

$$
f(x, y)=\left\lfloor\prod_{(s, t) \in S_{x y}} g(s, t)\right]^{\frac{1}{m n}}
$$

\subsection{Harmonic Mean Filter}

In the harmonic mean method, the gray value of each pixel is replaced with the harmonic mean of gray values of the pixels in a surrounding region. The harmonic mean [16] is defined as

$$
f(x, y)=\frac{m n}{\sum_{(s, t) \in S_{x y}} \frac{1}{g(s, t)}}
$$

\subsection{Contra Harmonic Mean Filter}

The contra harmonic mean filter operation is given by the expression

$$
f(x, y)=\frac{\sum_{(s, t) \in S_{x y}} g(s, t)^{Q+1}}{\sum_{(s, t) \in S_{x y}} g(s, t)^{Q}}
$$

Where $\mathrm{Q}$ is called the order of the filter. This filter is well suited for reducing or virtually eliminating the effects of salt-and-pepper noise.

\subsection{Median Filter}

Order-statistics filters are based on ordering the pixels contained in the mask. Median filter comes under this class of filters. Median filter replaces the value of a pixel with the median value of the gray levels within the filter window [17] or mask. Median filters are very effective for impulse noise.

$$
f(x, y)=\underset{(s, t) \in S_{x y}}{\text { median }}\{g(s, t)\}(8)
$$

\subsection{Midpoint filter}

This filter computes the midpoint between the maximum and minimum values in the area encompassed by the filter. This filter works best for randomly distributed noise like Gaussian noise.

$$
f(x, y)=\frac{1}{2}\left\{\max _{(s, t) \in S_{x y}}\{g(s, t)\}+\min _{(s, t) \in S_{x y}}\{g(s, t)\}\right\rceil
$$

\section{PERFORMANCE MEASUREMENT PARAMETERS}

Consider an image of dimensions $\mathrm{M}$ and $\mathrm{N}$. If $\mathrm{f}(\mathrm{x}, \mathrm{y})$ is the original image and $\mathrm{g}(\mathrm{x}, \mathrm{y})$ is the distorted image then the various measurement parameters are described as follows.

\subsection{Mean Square Error (MSE)}

The MSE [19] is cumulative squared error between the compressed and the original image. It is calculated using

$$
M S E=\frac{1}{M N} \sum_{0}^{M-1} \sum_{0}^{N-1}|| f(x, y)-g(x, y)||
$$


4.2 Peak Signal to Noise Ratio (PSNR)

The PSNR is used to determine the ratio between the maximum power of a signal and power of corrupting noise. The formula of PSNR is given as

$P S N R=10 \log _{10}\left[\frac{M * N}{M S E}\right]$

4.3 Average Difference (AD)

The average difference is given by the formula $\mathrm{AD}=|f(x, y)-g(x, y)|$

\subsection{Maximum Difference (MD)}

The maximum difference is given by the formula

$$
\mathrm{MD}=\max |f(x, y)-g(x, y)|
$$

\subsection{Normalized Absolute Error (NAE)}

The normalized error is given by

$$
y=N A E=\frac{\sum_{x=1}^{M} \sum_{y=1}^{N}(f(x, y) * g(x, y))}{\sum_{x=1}^{M} \sum_{y=1}^{N}(f(x, y))^{2}}
$$

\subsection{Structural Content (SC)}

$\mathrm{SC}$ is correlation based measure and measures the similarity between two images. It is given by the equation

$$
S C=\frac{\sum_{i=1}^{M} \sum_{j=1}^{N}(y(i, j))^{2}}{\sum_{i=1}^{M} \sum_{j=1}^{N}(x(i, j))^{2}}(15)
$$

\section{SIMULATION RESULT AND ANALYSIS}

Simulation has been run on MATLAB using gray scale image 'lena.bmp' of size 512 × 512 as a test image shown in Fig 1

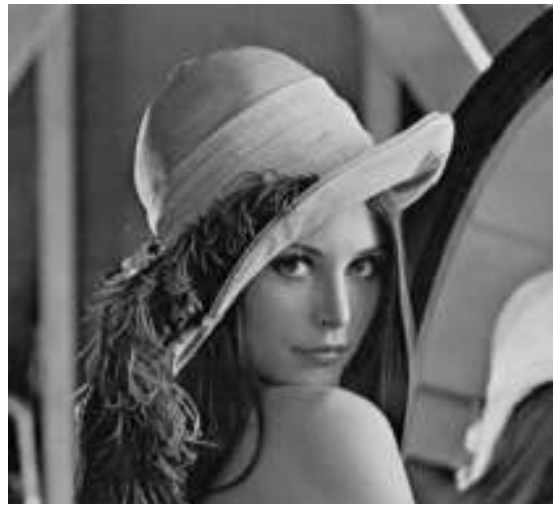

Fig. 1.Test Image Lena

For each and every noise all seven filters are applied in sequence and parameters are distinguished which are given below in the table. Table 1 shows the different measurement parameters after applying all the filters for Poisson noise.
Table.1 POISSON NOISE

\begin{tabular}{lllllll}
\hline $\begin{array}{l}\text { FILTE } \\
\text { R }\end{array}$ & MSE & $\begin{array}{l}\text { PSN } \\
\text { R }\end{array}$ & AD & MD & NAE & SC \\
\hline $\begin{array}{l}\text { Arithm } \\
\text { etic }\end{array}$ & 114. & 27.5 & 0.4 & 112 & 0.062 & 1.0 \\
Filter & & 49 & 13 & & & 073 \\
$\begin{array}{l}\text { Geome } \\
\text { tric }\end{array}$ & 24.4 & 34.2 & 3.1 & 182 & 0.031 & 0.9 \\
$\begin{array}{l}\text { Filter } \\
\text { Harmo }\end{array}$ & 31.1 & 34 & 02 & & & 991 \\
nic & 5 & 96 & 3.8 & 182 & 0.039 & 1.0 \\
Filter & & & & & & 017 \\
Contra & 252. & 24.1 & 98. & 252 & 0.996 & 159 \\
- & 15 & 1 & 72 & & & .37 \\
Harmo & & & & & & \\
nic & & & & & & \\
$\begin{array}{l}\text { Filter } \\
\text { Media }\end{array}$ & 19.7 & 35.1 & 2.5 & 135 & 0.025 & 0.9 \\
n Filter & 9 & 6 & 6 & & 9 & 983 \\
$\begin{array}{l}\text { Max } \\
\text { and }\end{array}$ & 79.6 & 29.1 & 10. & 182 & 0.103 & 1.0 \\
Min & & 1 & 26 & & & 192 \\
$\begin{array}{l}\text { Filter } \\
\text { Mid- }\end{array}$ & 16.2 & 36.0 & 2.1 & 89 & 0.021 & 0.9 \\
Point & 437 & 2 & 378 & & 6 & 975 \\
Filter & & & & & & \\
\hline
\end{tabular}

Table 2 shows the different measurement parameters after applying all the filters for Multiplicative noise.

Table.2 MULTIPLICATIVE NOISE

\begin{tabular}{lllllll}
\hline FILTE & MSE & PSN & A & MD & NAE & SC \\
R & & R & D & & & \\
\hline Arithm & 32.4 & 33.0 & 3. & 111 & 0.03 & 0.9 \\
etic & 189 & 228 & 94 & & 98 & 975 \\
Filter & & & 6 & & & \\
Geome & 51.9 & 30.9 & 5. & 182 & 0.05 & 0.9 \\
tric & 361 & 761 & 32 & & 38 & 987 \\
Filter & & & 5 & & & \\
Harmo & 68.6 & 29.7 & 6. & 182 & 0.07 & 1.0 \\
nic & 100 & 669 & 99 & & 06 & 006 \\
Filter & & & 4 & & & \\
Contra & 252. & 24.1 & 98 & 252 & 0.99 & 155 \\
- & 150 & 142 & .6 & & 65 & .07 \\
Harmo & & & 97 & & & \\
nic & & & & & & \\
Filter & & & & & & \\
Media & 50.9 & 31.0 & 5. & 135 & 0.05 & 0.9 \\
n Filter & 206 & 619 & 16 & & 21 & 983 \\
& & & 1 & & & \\
Max & 80.2 & 29.0 & 10 & 182 & 0.10 & 1.0 \\
and & 308 & 874 & .3 & & 43 & 192 \\
Min & & & 34 & & & \\
Filter & & & & & & \\
Mid- & 16.5 & 35.9 & 2. & 89 & 0.02 & 0.9 \\
Point & 379 & 460 & 17 & & 19 & 975 \\
Filter & & & 31 & & & \\
\hline & & & & & & \\
\hline
\end{tabular}



ISSN : 2248-9622, Vol. 7, Issue 7, ( Part -1) July 2017, pp.35-39

Table 3 shows the different measurement parameters after applying all the filters for Exponential noise.

\begin{tabular}{lllllll}
\multicolumn{1}{c}{ Table.3 } & \multicolumn{5}{c}{ EXPONENTIAL NOISE } \\
\hline FILTE & MSE & PSN & A & MD & NAE & SC \\
R & & R & D & & & \\
\hline Arithm & 7.91 & 39.1 & 0. & 102 & 0.00 & 0.9 \\
etic & 38 & 470 & 93 & & 95 & 989 \\
Filter & & & 6 & & & \\
Geome & 5.88 & 40.4 & 0. & 112 & 0.00 & 0.9 \\
tric & 60 & 326 & 70 & & 71 & 975 \\
Filter & & & 1 & & & \\
Harmo & 9.37 & 38.4 & 1. & 157 & 0.01 & 0.9 \\
nic & 86 & 094 & 14 & & 15 & 980 \\
Filter & & & 3 & & & \\
Contra & 251. & 24.1 & 98 & 252 & 0.99 & 125 \\
- & 964 & 174 & .5 & & 45 & .28 \\
Harmo & & & 01 & & & \\
nic & & & & & & \\
Filter & & & & & & \\
Media & 4.33 & 41.7 & 0. & 135 & 0.00 & 0.9 \\
n Filter & 44 & 615 & 58 & & 59 & 970 \\
& & & 8 & & & \\
Max & 70.7 & 29.6 & 9. & 182 & 0.09 & 1.0 \\
and & 486 & 336 & 17 & & 26 & 149 \\
Min & & & 16 & & & \\
Filter & & & & & & \\
Mid- & 15.9 & 36.1 & 2. & 89 & 0.02 & 0.9 \\
Point & 580 & 010 & 10 & & 13 & 975 \\
Filter & & & 84 & & & \\
\hline
\end{tabular}

Table 4 shows the different measurement parameters after applying all the filters for Erlang noise.

Table.4 ERLANG NOISE

\begin{tabular}{lllllll}
\hline FILTE & MSE & PSN & A & MD & NAE & SC \\
R & & R & D & & & \\
\hline Arithm & 1.47 & 46.4 & 0. & 81 & 0.00 & 0.9 \\
etic & 51 & 425 & 17 & & 18 & 997 \\
Filter & & & 3 & & & \\
Geome & 0.60 & 50.3 & 0. & 97 & 0.00 & 0.9 \\
tric & 50 & 136 & 06 & & 0634 & 990 \\
Filter & & & 2 & & 02 & \\
Harmo & 1.70 & 45.8 & 0. & 131 & 0.00 & 0.9 \\
nic & 48 & 142 & 19 & & 20 & 981 \\
Filter & & & 5 & & & \\
Contra & 251. & 24.1 & 98 & 252 & 0.99 & 106 \\
- & 671 & 225 & .1 & & 14 & .41 \\
Harmo & & & 98 & & & \\
nic & & & & & & \\
Filter & & & & & & \\
Media & 0.42 & 51.8 & 0. & 135 & 0.00 & 0.9 \\
n Filter & 91 & 054 & 05 & & 0531 & 987 \\
& & & 2 & & 11 & \\
Max & 42.9 & 31.8 & 5. & 175 & 0.05 & 1.0 \\
and & 052 & 057 & 61 & & 67 & 041 \\
Min & & & 72 & & & \\
\hline
\end{tabular}

\begin{tabular}{lllllll}
\hline Filter & & & & & & \\
Mid- & 15.9 & 36.1 & 2. & 89 & 0.02 & 0.9 \\
Point & 580 & 010 & 10 & & 13 & 975 \\
Filter & & & 84 & & & \\
\hline
\end{tabular}

\section{CONCLUSION}

On seeing the parameters shown in the above tables we can conclude that which filter will be best for removing respective noise. This conclusion is stated below in the Table 5 .

Table.5 FILTERS TO BE CHOSEN FOR DIFFERENTNOISES

\begin{tabular}{ll}
\hline NOISES & FILTERS \\
\hline Poisson Noise & Mid-Point Filter \\
Erlang Noise & Geometric Mean Filter \\
Multiplicative noise & Mid-Point Filter \\
Exponential Noise & Median Filter \\
\hline
\end{tabular}

\section{REFERENCES}

[1] R.C. Gonzalez and R.E.Woods"Digital Image Processing

[2] Suresh Kumar, Papendra Kumar, Manoj Gupta, Ashok Kumar Nagawat, "Performance Comparison of Median and Wiener Filter in Image De-noising", International Journal of Computer Application, Vol.12 - No.4, November 2010.

[3] C.Saravanan, R. Ponalagusamy"Gaussian Noise Estimation Technique for Gray Scale Images Using Mean Value". Journal of Theoretical and Applied Information technology. 2005-2007.

[4] S. P. Awate and R. T. Whitaker, "Higherorder image statistics for unsupervised, information-theoretic, adaptive, image filtering," in Proc IEEE Comput. Soc. Conf. Comput. Vis. Pattern Recognit., vol. 2. Jun. 2005, pp. 44-51.

[5] A. Buades, B. Coll, and J.-M. Morel, "A non-local algorithm for image denoising," in Proc. IEEE Comput. Soc. Conf. Comput. Vis. Pattern Recognit., vol. 2. Jun. 2005, pp. $60-65$

[6] M. Lebrun, “An analysis and implementation of the BM3D image denoising method," Image Process. On Line, vol. 2, pp. 175-213,Aug. 2012

[7] P. Blomgren and T. F. Chan, "Color TV: Total variation methods for restoration of vector-valued images," IEEE Trans. Image Process, vol. 7, no. 3, pp. 304-309, Mar. 1998.

[8] M. Lebrun, M. Colom, and J. M. Morel, "The noise clinic: A universal blind denoising algorithm," in Proc. IEEE Int. 
Conf. Image Process, Oct. 2014, pp. 2674 2678.

[9] T. Batard and M. Berthier, "Spinor Fourier transform for image processing," IEEE J. Sel. Topics Signal Process., vol. 7, no. 4, pp. 605-613, Aug. 2013.

[10] A. Levin and B. Nadler, "Natural image denoising: Optimality and inherent bounds," in Proc. IEEE Int. Conf. Comput. Vis. Pattern Recognit., vol. 2. Jun. 2011, pp. 2833-2840.

[11] M. Lysaker, S. Osher, and X.-C. Tai, "Noise removal using smoothed normals and surface fitting," IEEE Trans. Image Process., vol. 13, no. 10, pp. 1345-1357, Oct. 2004.

[12] S. Osher, M. Burger, D. Goldfarb, J. Xu, and W. Yin, "An iterative regularization method for total variation-based image restoration," Multiscale Model. Simul., vol. 4, no. 2, pp. 460-489, 2005.

[13] T. Rahman, X.-C. Tai, and S. Osher, "A tvstokes denoising algorithm," in Scale Space and Variational Methods in Computer Vision (Lecture Notes in Computer Science), vol. 4485. Berlin, Germany, Springer Verlag, 2007, pp. 473-483. 